\title{
A rare case of perivascular epithelioid cell tumor (PEComa) of the pancreas diagnosed by endoscopic ultrasound $\square$
}

\section{다)(1) $(\odot$}

\author{
Authors \\ Institutions \\ 1 Klinik und Poliklinik für Innere Medizin II, Klinikum \\ rechts der Isar der Technischen Universität München, \\ Munich, Germany \\ 2 Institut für Allgemeine Pathologie und Pathologische \\ Anatomie, Technische Universität München, Munich, \\ Germany \\ 3 Klinik und Poliklinik für Chirurgie, Klinikum rechts der \\ Isar der Technischen Universität München, Munich, \\ Germany
}

J. D. Ulrich ${ }^{1}$, K. Specht ${ }^{2}$, A. M. Schlitter ${ }^{2}$, G. O. Ceyhan ${ }^{3}$, M. Quante ${ }^{1}$, R. M. Schmid ${ }^{1}$, C. Schlag ${ }^{1}$

submitted 4.1.2019

accepted after revision 8.7.2019

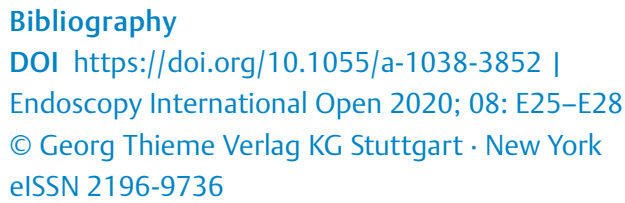

Corresponding author

Christoph Schlag, MD, Klinik und Poliklinik für Innere Medizin II, Klinikum rechts der Isar der Technischen Universität München, 81675 Munich, Germany

Fax: +49-89-41404871

christoph.schlag@mri.tum.de

\begin{abstract}
A 49-year-old woman consulted her general practitioner (GP) regarding epigastric pain that she had experienced for 2 months. Physical examination and laboratory results were unremarkable. An abdominal ultrasound indicated a solid pancreatic tumor, which was confirmed on subsequent CT and MRI. Endoscopic ultrasound (EUS) showed a well-defined heterogeneous, predominantly hypoechoic mass in the pancreatic body, so a neuroendocrine tumor (NET) was suspected. However, EUS-guided fine-needle aspiration (EUS-FNA) was performed and based on (immuno-)histochemical findings, the extremely rare diagnosis of a perivascular epithelioid cell tumor (PEComa) of the pancreas was made. Due to the malignant potential of pancreatic PEComas, laparoscopic left-sided pancreatectomy was performed. We present a case diagnosed by preoperative EUSFNA highlighting the clinical and endosonographic features which help to distinguish it from its most important differential diagnosis, neuroendocrine tumors (NETs) of the pancreas.
\end{abstract}

of note in her family history. She was a smoker (30 pack-years), consumed little alcohol, and took proton pump inhibitors (PPIs) when required due to the above-mentioned pain; however, she did not experience any symptom relief.

Physical examination and routine laboratory results did not show any pathological findings apart from a mild iron deficiency anemia most likely due to the hypermenorrhea. Abdominal ultrasound performed by the GP showed a round, defined, $25 \mathrm{~mm}$ hypoechogenic mass in the pancreatic body without dilation of the pancreatic duct which was confirmed on subsequent $C T$ and MRI ( $\vee$ Fig. 1). As a pancreatic neuroendocrine tumor (NET) was suspected, Chromogranin A serum level was measured showing only a slight elevation of $145 \mu \mathrm{g} / \mathrm{L}$ (reference $<102 \mu \mathrm{g} / \mathrm{L})$.

We performed endoscopic ultrasound (EUS) (with Pentax radial scanner, Pentax EG36704URK, Pentax Europe, Hamburg, 


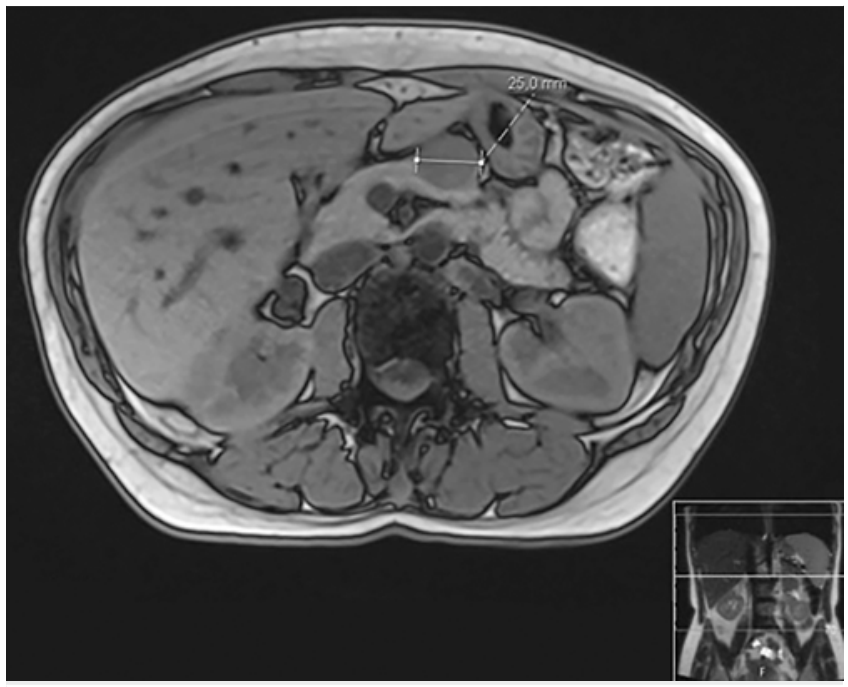

- Fig. 1 T1-weighted MRI of the abdomen showing a hypointense mass in the body of the pancreas.

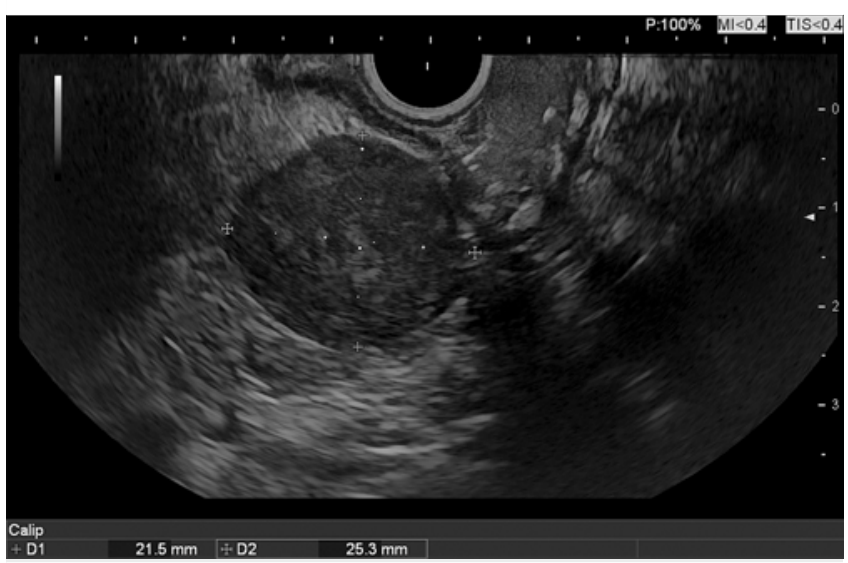

Fig. 2 Endoscopic ultrasound (EUS) showing a well-defined heterogeneous, predominantly hypoechoic mass with lateral shadowing in the pancreatic body measuring $25 \times 20 \mathrm{~mm}$ without dilation of the pancreatic duct.

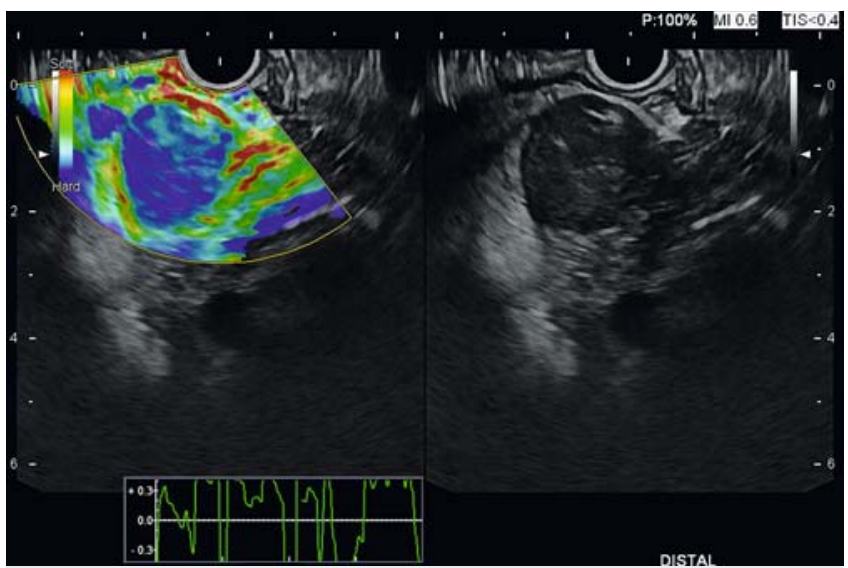

- Fig. 3 Elastography indicated that the lesion had a rather hard (=blue) tissue appearance.
Germany) which confirmed the homogenous appearance of the pancreatic tissue but with a well-defined heterogeneous, predominantly hypoechoic mass with lateral shadowing in the pancreatic body and measuring $25 \times 20 \mathrm{~mm}$ without dilation of the pancreatic duct ( $>$ Fig. 2, > Video 1). EUS elastography indicated that the lesion had a rather hard (=blue) tissue appearance ( Fig. 3, V Video 1). With B-mode (power)-Doppler, the hypervascularity of the lesion could already be suspected. Contrast Enhanced Low Mechanical Index Endosonography (CELMI-EUS) (SonoVue, Bracco, Italy) showed a clear and long-lasting hyperperfusion with late "wash-out" of the lesion ( Video 1 ).

EUS-Guided fine-needle aspiration (EUS-FNA) with a 22 gauge needle (EchoTip Ultra, Cook Medical, Limerick, Ireland) was performed using the fanning technique with slow-pull of the stylet and the tissue material obtained was fixed in CytoRich Red (Fisher Scientific, Schwerte, Germany) solution for further cell block analysis.

Hematoxylin and eosin (H\&E) staining revealed cells consisting of sheets of uniform, epithelioid-spindle shaped cells with abundant granular eosinophilic cytoplasm and distinct prominent nucleoli ( $\mathbf{F i g} \mathbf{4 b}$ ). Additional immunohistochemical stains were also performed. The tumor cells showed positivity for smooth muscle markers desmin and actin as well as for melanocytic markers HMB-45 ( $\triangleright$ Fig.4c) and Melan-A ( but negativity for synaptophysin, chromogranin $A$, cytokeratin (panCK, CK20, CK7), CD-117, and S-100.

Based on these findings, the diagnosis of a perivascular epithelioid cell tumor (PEComa) of the pancreas was made and other potential diagnoses such as NET, metastasis, or pancreatic cancer could be excluded. Due to the malignant potential of pancreatic PEComas, laparoscopic left-sided pancreatectomy with splenectomy was performed. The resected specimen showed a $2.5 \mathrm{~cm} \times 2.5 \mathrm{~cm} \times 2.3 \mathrm{~cm}$ well-circumscribed encapsulated spherical tumor with a homogeneous light brown cut surface, surrounded by normal lobular pancreatic parenchyma with tumor-free margins. Furthermore, spleen and resected

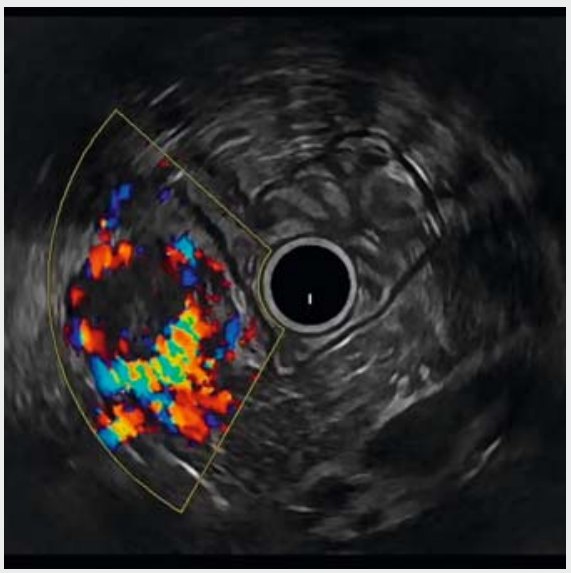

$\checkmark$ Video 1 PEComa in EUS. Doppler imaging, elastography, contrast-enhanced EUS, and contrast enhanced low mechanical index endosonography (CELMI-EUS). 

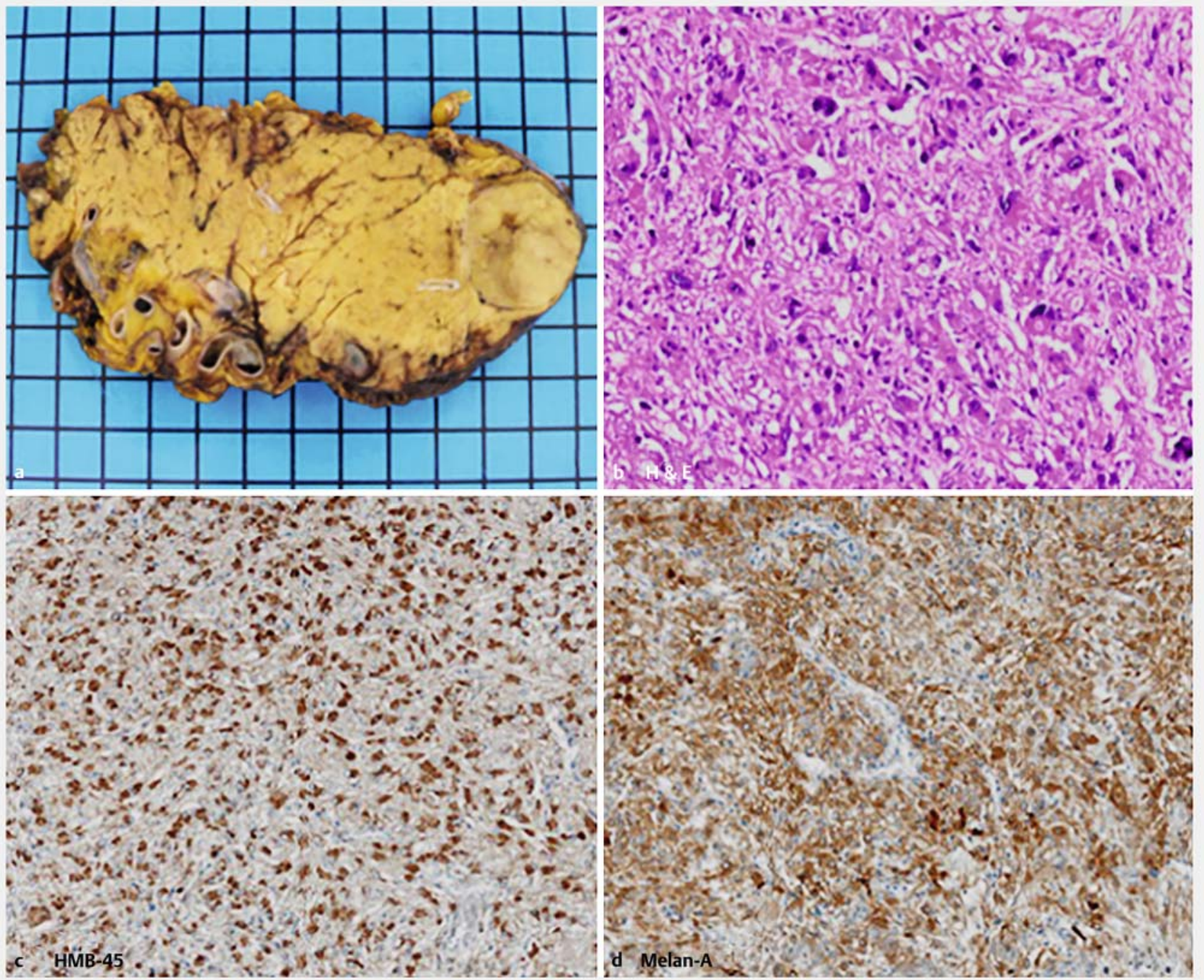

- Fig. 4 a Resected specimen showed a well-circumscribed encapsulated tumor, surrounded by normal lobular pancreatic parenchyma. b H\&E staining revealed cells consisting of sheets of uniform epithelioid-spindle shaped cells with abundant granular eosinophilic cytoplasm and distinct prominent nucleoli. The tumor cells showed positivity for melanocytic markers HMB-45 (c) and Melan-A (d).

lymph nodes were free from metastases. Histological and immunohistological analysis of the resected specimen revealed the same features as already shown by previous EUS-FNA ( Fig.4).

\section{Discussion}

Perivascular epithelioid cell tumors (PEComas), first described by Bonetti et al. in 1992 [1], are rare mesenchymal cell neoplasms that can occur in every part of the human body including soft tissue, bone, abdominopelvic sites, and retroperitoneal sites such as the uterus or kidney. In 1996, Zamboni et al. reported on the occurrence of PEComas in the pancreas and introduced the term "sugar tumor" due to the clear cell cytoplasm of the perivascular epithelioid cells, which are rich in glycogen in this tumor [2]. Histopathologically, these tumors are characterized by cells with a spindle or epithelioid shape typi- cally spread around blood vessels. The characteristic immunohistochemical profile shows positivity for melanocytic markers (such as HMB-45 and Melan-A) and smooth muscle markers (such as actin and desmin) [3].

To our knowledge, at the present time, only 21 cases of primary PEComa of the pancreas have been reported since the first description in 1996 by Zamboni et al. [2], and most recently in the report by Zhang et al. [4]. These tumors can arise at any age, can affect either sex, but as in our case, seem to have a predilection for middle-aged women. Patients mostly present with fluctuant abdominal pain, swelling in the right upper quadrant, and occasionally diarrhea, though in some cases, patients are asymptomatic. There are no specific laboratory tests to screen for PEComas [4]. In our case, the mild anemia was probably caused by the hypermenorrhea, and the slightly elevated Chromogranin A most likely due to occasional intake of PPIs [5]. 
In all image modalities (abdominal US, EUS, CT, MRI), pancreatic PEComas are usually characterized by well-circumscribed, oval- to round-shaped masses with surrounding normal pancreatic tissue, lack of infiltration, and signs of arterial hypervascularity when color Doppler or contrast-enhanced modes are used [6]. As shown in our case, elastography demonstrated a hard tissue lesion with lateral shadowing, consistent with an encapsulated tumor. All of these imaging findings mimic pancreatic NETs but can already help to differentiate it from pancreatic adenocarcinoma, where the appearance is that of a poorly defined hypodense mass with infiltrating growth [6]. Furthermore, we observed a very long-lasting contrast enhancement in CELMI-EUS which might even help to differentiate this lesion from pancreatic NETs.

Other lesions to keep on the list of differentials include pseudopapillary neoplasm, gastrointestinal stroma tumor (GIST), acinar cell carcinoma of the pancreas, and metastasis of clear cell renal cell carcinoma or melanoma, though pancreatic NET remains the most important alternative diagnosis to exclude. European guidelines recommend enucleation at surgery for suspected NET with a solid pancreatic lesion $>2 \mathrm{~cm}$ [7]. The present case impressively demonstrates the advantages of EUS-FNA, combining the best imaging modality with the ability to obtain a tissue diagnosis, showing that a suspected NET can be a PEComa on histopathology. Thus, exact tissue diagnosis before further treatment seems to be most reasonable as it influences clinical management.

Reviewing the literature, in 14 of 22 cases including our case a preoperative EUS-FNA was performed. In only six, the diagnosis "PEComa" was established preoperatively following the use of immunohistochemical staining for HMB-45. The other eight cases were either nondiagnostic or misinterpreted as carcinoma or neuroendocrine tumors $[4,8]$. Collins et al. noticed that, in these cases, the material obtained was only used for preparing a cytological smear. The material was very scant or nondiagnostic, in their understanding most likely due to extreme cohesion of PEComa cells to each other and the matrix [8]. Thus, the benefit of smear cytology in diagnosing solid pancreatic lesions such as PEComa seems questionable. For a definitive diagnosis, microscopic examination of a H\&E stained tissue cell block with the option to perform immunohistochemistry would be advised. Formalin fixed histology might be a good alternative but requires core tissue material which cannot always be obtained by EUS-guided tissue sampling.

The great majority of pancreatic PEComas are considered benign and the prognosis is relatively good with most patients remaining free from relapse and metastasis [4]. So far, to our knowledge, four primary malignant pancreatic PEComas have been reported. In two cases, liver metastases occurred, and in two cases the tumor showed malignant features such as infiltrating growth, necrosis, and size $>5 \mathrm{~cm}$, most recently reported by Zhang et al. In this case, adjuvant chemotherapy with a sarcoma regimen with epirubicin and ifosfamide seems to be effective [4].

In general, PEComas might have an association with tuberous sclerosis complex (TSC). However, the association between pancreatic PEComa and TSC remains vague, since there is only one described case of a women with TSC and pancreatic PEComa reported by Hartley et al. [9]. In our case, no signs of TSC were observed.

In conclusion, PEComa of the pancreas remains an extremely rare diagnosis of a solid pancreatic tumor with malignant potential. In EUS, which generally provides a very good image modality for diagnosing pancreatic lesions with high sensitivity and specificity, PEComas show very similar characteristics to NET, but in CELMI-EUS, a longer lasting contrast enhancement can be seen.

However, additional EUS-FNA with further adequate immunohistochemical processing seems reasonable to establish the right diagnosis to guide further therapy.

\section{Competing interests}

None

References

[1] Bonetti F, Pea M, Martignoni G et al. PEC and sugar. Am J Surg Pathol 1992; 16: 307-308

[2] Zamboni G, Pea M, Martignoni G et al. Clear cell "sugar" tumor of the pancreas: A novel member of the family of lesions characterized by the presence of perivascular epithelioid cells. Am J Surg Pathol 1996; 20: $722-730$

[3] Fletcher CDM, Bridge JA, Hogendoorn P et al. WHO classification of tumours of soft tissue and bone.4th edn. Geneva: WHO; 2013

[4] Zhang S, Chen F, Huang X et al. Perivascular epithelial cell tumor (PEComa) of the pancreas: A case report and review of literature. Medicine 2017; 96: e7050

[5] Giusti M, Sidoti M, Augeri C et al. Effect of short-term treatment with low dosages of the proton-pump inhibitor omeprazole on serum chromogranin A levels in man. Eur J Endocrinol 2004; 150: 299-303

[6] Tirumani SH, Shinagare AB, Hargreaves ] et al. Imaging features of primary and metastatic malignant perivascular epithelioid cell tumors. Am J Roentgenol 2014; 202: 252-258

[7] Öberg K, Knigge U, Kwekkeboom D et al. Neuroendocrine gastro-entero-pancreatic tumors: ESMO Clinical Practice Guidelines for diagnosis, treatment and follow-up. Ann Oncol 2012; 23: vii124-vii130

[8] Collins K, Buckley T, Anderson K et al. Perivascular epithelioid cell tumor (PEComa) of pancreas diagnosed preoperatively by endoscopic ultrasound-guided fine-needle aspiration: A case report and review of literature. Diagn Cytopathol 2017; 45: 59-65

[9] Hartley CP, Kwiatkowski DJ, Hamieh L et al. Pancreatic PEComa is a novel member of the family of tuberous sclerosis complex-associated tumors: case report and review of the literature. Virchows Archiv 2016; 469: 707-710 